\title{
DESIGN READINESS OF MULTI-MATERIAL CONCEPTS: MANUFACTURING AND JOINING TECHNOLOGY INTEGRATED EVALUATION OF CONCEPT MATURITY LEVELS USING CARDINAL COEFFICIENTS
}

\author{
S. Revfi ${ }^{1, \otimes}$, J. Wilwer ${ }^{1}$, K. Behdinan ${ }^{2}$ and A. Albers ${ }^{1}$ \\ ${ }^{1}$ Karlsruhe Institute of Technology, Germany, ${ }^{2}$ University of Toronto, Canada \\ $\square$ sven.revfi@kit.edu
}

\begin{abstract}
Maturity levels of components in early phases of product development are often assessed with Technology Readiness Levels. However, developing Multi-Material-Design (MMD) concepts for lightweight design, not only the manufacturability of the individual components is decisive, but also their joinability with each other and their integration into the rest system. This paper presents an approach for the evaluation of maturity levels of MMD concepts on the basis of cardinal coefficients considering a time forecast of the manufacturing and joining technologies required in the concept.
\end{abstract}

Keywords: design management, design readiness, lightweight design, multi-material design, risk management

\section{Introduction}

Bringing new products to the market secures economic success for most companies. This requires either the fulfilment of new product functions or the same functions through new technical inventions (e. g. with new technologies). Both possibilities can lead to an increasing product complexity. As a result, product development processes become increasingly complex and can only be handled by multidisciplinary teams. By using the latest technologies for new innovative products, the product development teams try to achieve a competitive advantage in the market. However, the use of new technologies in a technical system leads to implementation risks due to uncertainties. Uncertainties concerning the maturity of a technology can result in budget and schedule risks in the concept phase as well as in performance risks of the final product.

In the industrial sector, the use of different materials and manufacturing technologies within a concept raises the question of how well the corresponding technologies needed for individual components are already matured. In addition, it is imperative to assess whether the components can be joined together or whether they can be joined with the rest system.

The use of methods within technology management, which can assess the maturity of a technology, has the potential to evaluate such risks (Mankins, 2009a, 2009b). One way of assessing technological maturity was provided by the National Aeronautics and Space Administration (NASA) through the Technology Readiness Level (TRL). In this method, technological maturity is subdivided into nine levels (Table 1). 
Table 1. TRL and corresponding definition (Mankins, 1995)

\begin{tabular}{|c|l|}
\hline TRL & Definition \\
\hline $\mathbf{1}$ & Basic principles observed and reported \\
\hline $\mathbf{2}$ & Technology concept and/or application formulated \\
\hline $\mathbf{3}$ & Analytical and experimental critical function and/or characteristic proof of concept \\
\hline $\mathbf{4}$ & Component and/or breadboard validation in laboratory environment \\
\hline $\mathbf{5}$ & Component and/or breadboard validation in relevant environment \\
\hline $\mathbf{6}$ & System/subsystem model or prototype demonstration in a relevant environment (ground or space) \\
\hline $\mathbf{7}$ & System prototype demonstration in operational environment \\
\hline $\mathbf{8}$ & Actual system completed and qualified through test and demonstration (ground or space) \\
\hline $\mathbf{9}$ & Actual system proven through successful mission operations \\
\hline
\end{tabular}

However, a technology maturity assessment with the TRL is only possible to a limited extent. Reasons for this are that an uncertainty in the future development of maturity and the influence on interactions with other technologies in the overall system cannot be assessed. In order to include the interactions of individual components for the evaluation of an overall system maturity, a so-called Integration Readiness Level (IRL) was defined similar to the TRL (Table 2). (Sauser et al., 2009)

Table 2. IRL and corresponding definition (Gove et al., 2010)

\begin{tabular}{|c|l|}
\hline IRL & Definition \\
\hline $\mathbf{1}$ & $\begin{array}{l}\text { An Interface between technologies has been identified with sufficient detail to allow characterization } \\
\text { of the relationship }\end{array}$ \\
\hline $\mathbf{2}$ & Some level of specification to characterize an interaction between technologies has been achieved \\
\hline $\mathbf{3}$ & There is compatibility between technologies to orderly and efficiently integrate and interact \\
\hline $\mathbf{4}$ & There is enough detail in the quality and assurance of the integration between technologies \\
\hline $\mathbf{5}$ & $\begin{array}{l}\text { There is enough control between technologies necessary to establish, manage and terminate the } \\
\text { integration }\end{array}$ \\
\hline $\mathbf{6}$ & $\begin{array}{l}\text { The integration technologies can accept, translate and structure information for its intended } \\
\text { application }\end{array}$ \\
\hline $\mathbf{7}$ & The integration of technologies has been verified and validated with enough detail to be actionable \\
\hline $\mathbf{8}$ & $\begin{array}{l}\text { Actual integration completed and mission qualified through test and demonstration in the system } \\
\text { environment }\end{array}$ \\
\hline $\mathbf{9}$ & Integration is mission proven through successful mission operations \\
\hline
\end{tabular}

The combination of TRL and IRL has made it possible to define a System Readiness Level (SRL) which, depending on the choice of individual technologies and their interoperability with each other, determines the maturity of an entire system (Sauser et al., 2009). As products are developed according to the model of PGE - Product Generation Engineering (Albers et al., 2017) - in generations, the evaluation of the integration possibility is of decisive importance. The PGE model is based on the observation that only certain components of a product are newly developed (by principle or embodiment variations). The remaining components are carried over from the existing product generation on the market (or from other elements in the reference system) by means of carryover variations. Therefore, the evaluation of the integrability of the newly developed subsystems into an existing system is of crucial importance.

Austin and York (2015) present an example of calculating the System Readiness Assessment based on the identified TRLs and SRLs and propose an SRL Translation Model as a result. However, this approach, like that one of Sauser et al. (2009), is also based on the assumption that the TRL levels can be used for mathematical calculations.

But, TRL and IRL values are ordinal values. These merely indicate a certain trend in the product development process, but do not indicate the effort required to achieve the respective degree of maturity or the effort needed for future increase of the TRL level. As a result, the numbers could be replaced (e. g. by letters) at any time. Due to the ordinality of the TRL and IRL, the numerical values cannot be used in mathematical calculations (Fahimian and Behdinan, 2017). 
For the first time, Conrow (2011) succeeded determining cardinal TRL values using the Analytical Hierarchy Process (AHP) (Saaty, 1990). The comparative matrix used in the AHP to determine technological maturity was developed on the basis of qualitative expert opinions. However, from this work it was not clear which criteria were used to determine the qualitative expert opinion, nor which scale was used to measure a technology maturity. As a consequence, Fahimian and Behdinan (2017) presented a possibility to calculate cardinal coefficients for the determination of a TRL using quantitative data. The criterion was the time taken by a technology to move up to the next TRL. This time is called the maturity time.

With the methods presented, no integration of subsystems into an existing rest system with joining technologies depending on the temporal development of the used technologies can be modelled.

\section{Research objective}

The evaluation of maturity levels of concept ideas can support product development teams in concept selection. For this, a mutual comparability of the concepts is crucial. In lightweight design, the integrated consideration of the associated joining technologies plays a decisive role especially for Multi-Material-Design (MMD) concepts that pursue a requirements and load-case oriented combination of materials. However, as products are developed in generations, attention must not only be paid to the joining of individual materials and components to a new (sub)system, but also to the integration of the newly developed subsystem into the existing rest system. This leads to a big challenge in the objective evaluation and comparability of maturity levels of MMD concepts.

In the following, a Design Readiness Level (DRL) for objectifying MMD concept maturity level evaluations is presented. For this purpose, the maturity level assessment of manufacturing processes on the basis of TRL cardinal coefficients is extended by a time forecast, transferred to IRL as the assessment of joining technologies and combined with the SRL approach proven in the literature. Including the time forecast in the calculation of TRL and IRL cardinal coefficients makes maturity levels of different concepts comparable in terms of their individual time to reach series maturity. Therefore, the aim of this procedure is an objective design readiness assessment for MMD concepts with regard to the predicted future development of the manufacturing and joining technologies used in the concept.

\section{Method}

To be able to consider the factor time within the TRL as a criterion for a technology's maturity, the AHP is used. The comparison matrix A correlates the ratios of the maturity times of different TRLs and is defined in Equation (1). The approach for the determination of cardinal coefficients is discussed in detail in the work of Fahimian and Behdinan (2017). As indicated by the value range of the running index $i$, the TRL metric with nine levels developed by NASA (Mankins, 1995) does not necessarily have to be used.

$$
\mathbf{A}_{i \times j}=\left[\begin{array}{ccc}
\frac{t_{T R L 1}}{t_{T R L 1}} & \cdots & \frac{t_{T R L 1}}{t_{T R L i}} \\
\vdots & \ddots & \vdots \\
\frac{t_{T R L i}}{t_{T R L 1}} & \cdots & \frac{t_{T R L i}}{t_{T R L i}}
\end{array}\right], i \in \mathbb{N}
$$

Since, by definition, TRL 1 is achieved by the mere observation of a scientific principle, a maturity time cannot be described. Therefore, the assumption was made that the maturity time to reach TRL 1 is equal to one year. The consequences of this assumption and of AHP modelling for the calculation of the TRL cardinal coefficients are discussed in detail by Fahimian and Behdinan (2017) and adopted for this paper. Here, it is noted that the maturity time for TRL 1 should be correctly set to zero. However, this leads to invalid mathematical calculations due to the division by zero. An important characteristic of the cardinal coefficients determined by AHP is that their sum is always 1, irrespective of the chosen scale. This characteristic is essential for the interpretation of TRL cardinal coefficients and core of the developed method. For a technology with a TRL that is currently 3 (out of 9), for example, using the AHP results in three cardinal coefficients with a sum 
equal to 1 . For a technology with TRL 9 (of 9), there are nine cardinal coefficients, also with a sum equal to 1. The consequence of this characteristic is that the AHP does not provide comparable values for technologies with different ordinal TRL numbers.

However, within the concept phase of the MMD, it cannot be assumed that all technologies planned to be used within a concept are of the same maturity level and thus differ in their ordinal TRL numbers. This restricts the interpretability of the cardinal coefficients for the respective technologies.

In order to assess the impact of manufacturing or joining technologies still in development on the maturity level of MMD concept ideas, it is important that TRL and IRL cardinal coefficients are correctly interpreted and comparable with each other. This is explained using two technologies for which TRL cardinal coefficients were determined by Fahimian and Behdinan (2017). For the two NASA technologies "Non-destructive Evaluation" and "Direct To", the TRL cardinal coefficients were calculated on the basis of the AHP. The values shown in Table 3 are the result.

Table 3. TRL cardinal coefficients for two NASA's aeronautic projects (Fahimian and Behdinan, 2017)

\begin{tabular}{|c|c|c|}
\hline Ordinal TRL & $\begin{array}{l}\text { Non- } \\
\text { destructive } \\
\text { Evaluation }\end{array}$ & Direct To \\
\hline 1 & 0.05 & 0.12 \\
\hline 2 & 0.08 & 0.12 \\
\hline 3 & 0.14 & 0.13 \\
\hline 4 & 0.19 & 0.14 \\
\hline 5 & 0.24 & 0.24 \\
\hline 6 & 0.3 & 0.25 \\
\hline
\end{tabular}

In this case, the AHP was performed up to the ordinal TRL number of 6. Assuming that TRL 6 represents series maturity in this case, it becomes clear that the associated TRL cardinal coefficients (Non-destructive Evaluation $=0.3$; Direct To $=0.25$ ) are difficult to interpret without the information of the ordinal TRL and the progress of the associated TRL cardinal coefficients. Due to this fact, when interpreting maturity levels, the totality of the TRL cardinal coefficients should always be considered. This is particularly evident when looking at the ordinal TRL 5. Both technologies have the same TRL cardinal coefficient, which may suggest the same requested time for technologies to reach series maturity. But this is not the case as indicated by the TRL cardinal coefficient in TRL 6. Summing up this example, the cardinal coefficients can only be interpreted if they are in relation to all calculated cardinal values for one technology up to series maturity. Moreover, this gives a hint which further expenditure of time (and/or financial resources) is expected to develop the corresponding technology up to series maturity.

For the purpose of predicting the future technology development in the DRL, the previous progress of the technological maturity development is used for extrapolation. One possibility to calculate this time forecast is the prognosis function from MS Excel which uses the ETS algorithm. The quality of the forecast is decisive for the result of the method presented here. There is some uncertainty in this forecast (United States Government Accountability Office, 2011), which needs to be modelled. The investigation of different possibilities for modelling uncertainties is part of future research.

The extrapolated maturity times are used in the AHP to determine cardinal coefficients over the entire forecast period up to series maturity to specify the DRL. As the sum of the cardinal coefficients is always equal to 1 , the sum of the coefficients up to a considered ordinal TRL can now be interpreted as a progress towards reaching the predicted series maturity. For the two technologies "Nondestructive Evaluation" and "Direct To", a progress of $70 \%(0.05+0.08+0.14+0.19+0.24=0.7)$ and $75 \%$ would be achieved for the ordinal TRL 5 (still assuming that TRL 6 corresponds to series maturity). In the further course of this work, the progressions of the TRL and IRL are used.

In the following, the Design Readiness Level (DRL) of MMD concepts is introduced. It is based on the same equations as the SRL. The DRL comprises manufacturing (TRL) and associated joining technologies (IRL) and differs from the SRL, as it accounts for the predicted future developments of different technologies and in this way gives a comparable maturity of the current design. The IRL 
required to calculate the DRL is defined as the maturity of the joining technologies required to implement the concept, and is calculated similar to the TRL using the sum of its cardinal coefficients. In this context, joining technologies are defined as the permanent joining or any other kind of assembly of two or more components according to DIN (2003).

On the basis of the findings on the interpretation and comparability of TRL and IRL cardinal coefficients, the DRL for the assessment of the maturity levels of different MMD lightweight design concepts has been developed.

In a newly developed MMD concept consisting of $m$ components, in which different technologies for different components are used, the TRL is used in vector representation (Equation 2). The IRL between components, which in the case of MMD concepts represent the intended joining technologies, are represented as a matrix of the dimension $m \times m$ (Equation 3). Therefore, $I R L_{i j}(\mathrm{i}, \mathrm{j} \in 1,2, \ldots, \mathrm{m}$ ) represents the maturity of the joining technology between components $i$ and $j$ and thus equals $I R L_{j i}$. The hypothetical $I R L_{i i}$ is given as series maturity. If there is no interaction between component $\mathrm{i}$ and component $\mathrm{j}$, the value is set to zero. (Sauser et al., 2009)

$$
\begin{aligned}
T R L_{m \times 1} & =\left[\begin{array}{c}
T R L_{1} \\
T R L_{2} \\
\vdots \\
T R L_{m}
\end{array}\right] \\
\mathbf{I R L}_{m \times m} & =\left[\begin{array}{ccc}
I R L_{11} & \cdots & I R L_{1 m} \\
\vdots & \ddots & \vdots \\
I R L_{m 1} & \cdots & I R L_{m m}
\end{array}\right]
\end{aligned}
$$

The DRL vector is then calculated similar to the SRL vector by multiplying the IRL matrix and TRL vector (Equation 4) (Sauser et al., 2009).

$$
D R L_{m \times 1}=[\boldsymbol{I R L}]_{m \times m} *[T R L]_{m \times 1}
$$

The overall system maturity thus depends both on the individual maturity of the joining and manufacturing technologies to be used and on the architecture of the system under consideration. The calculation of the DRL is based on the findings of Sauser et al. (2009) and therefore the DRL values are normalized. The total system maturity is determined by the sum of all normalized DRL values divided by the number of components (Equation 5).

$$
D R L=\frac{\frac{D R L_{1}}{I_{1}}+\frac{D R L_{2}}{I_{2}}+\cdots+\frac{D R L_{m}}{I_{m}}}{m}, I_{i}=\text { Number of interactions on subsystem } i
$$

Another central issue is the integration of the new subsystem into the existing rest system. The joining technologies required for this integration must also be considered during concept development. The aim of determining a DRL taking the surrounding rest system into account is to uncover risks in the early phases of product development arising from uncertainties in the implementation of an MMD concept with regard to manufacturing and joining technologies.

Figure 1 (left) shows an exemplary race scooter gearbox system consisting of 17 components. Components 1 to 5 represent the subsystem "gearbox housing" for which new MMD concepts are exemplarily evaluated in the application example in section 4.

In order to ensure a manageable system, the surrounding rest system is simplified (Figure 1 (right)). This simplification is achieved by identifying similar working principles based on the $\mathrm{C} \& \mathrm{C}^{2}$ approach (Matthiesen et al., 2018). "Similar" in this case means that working surface pairs that are involved in functional fulfilment only differ quantitatively, but not conceptually. For example, this can be the case if several interfaces to the rest system are realized by cylindrical press fits, which only differ in their diameter and in the loads to be transferred. All components of the rest system which interact directly with the subsystem to be designed by means of similar working surface pairs are merged to a representative, aggregated substitute system. In the example shown in Figure 1 (left), this can be explained by the components D1 to D3 and E1 to E3. These components represent bearings which are pressed into the housing parts 3 and 5 and each support the shafts S1 to S3. Accordingly, these components are aggregated to the representative component K2 (Figure 1 (right)). As the components S1 
to S3 do not interact with the subsystem to be designed, they can be neglected within this aggregation. It should be noted that this procedure can only be used if neither the type of interaction nor a component in the rest system is to be changed. If a change of an interaction or component in the rest system by the developed concept is unavoidable, the subsystem boundary is to be extended in such a way that the required condition remains fulfilled. But this is also possible through fractal modelling with the ${\mathrm{C} \& \mathrm{C}^{2}}^{2}$ approach.
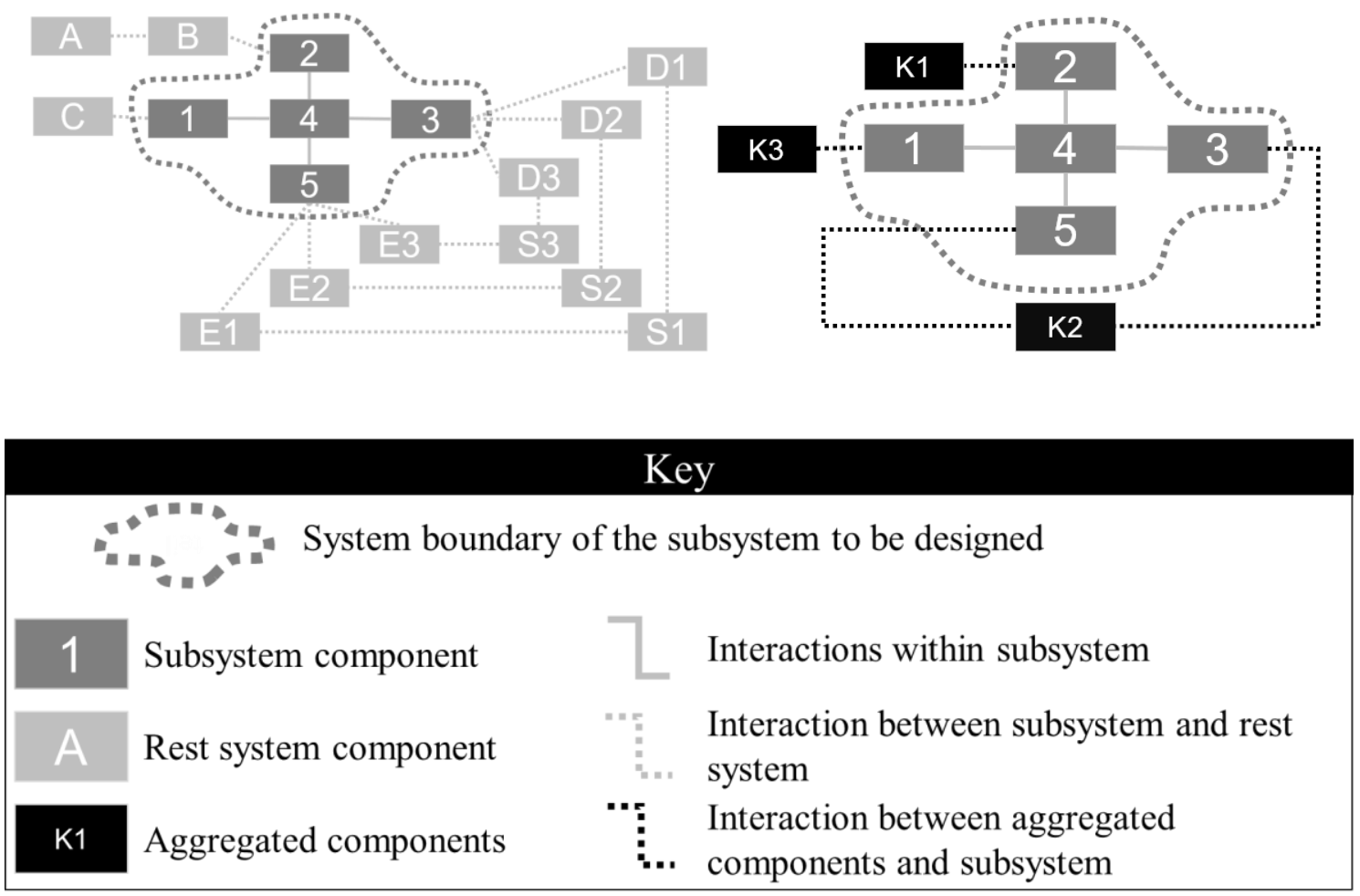

Figure 1. System diagram of a gearbox: full representation (left) and simplification (right)

Component aggregation reduces the calculation effort required to determine the DRL. If $\mathrm{k}$ aggregated subsystems are defined in total, these must be considered in the determination of the TRL and IRL in order to influence the overall system maturity. The TRL vector corresponds to the dimension $m+k \times$ 1 , the IRL matrix to the dimension $m+k \times m+k$.

Since the aggregated subsystems are already fully developed and will not be modified, their sums of the TRL cardinal coefficients are each given a 1. Potential interactions between aggregated components are not to be considered in the IRL matrix. Accordingly, their values in the IRL matrix are equal to 0 . This prevents a potentially complex rest system from having an influence on the calculation of the DRL.

\section{Application example}

The presented method is demonstrated using the simple system "gearbox housing" shown in Figure 2 even though the actual potential lies in the assessment of more complex systems by systematically supporting the identification of critical (sub)system interactions in conceptual design. The corresponding system architecture is given in Figure 1 (right).

Three fictitious MMD concepts (Table 4) are evaluated using new manufacturing and joining technologies. The sums of TRL and IRL cardinal coefficients required for the calculation of the DRL (including their forecasted development) were chosen exemplarily. The aim of the application example is not to provide reliable TRL and IRL values, but rather to clarify the procedure of the method. Furthermore, TRL and IRL of the same technologies differ from company to company depending on the individual level of knowledge and the planned application, which makes the provision of reliable values for this example even more difficult. 


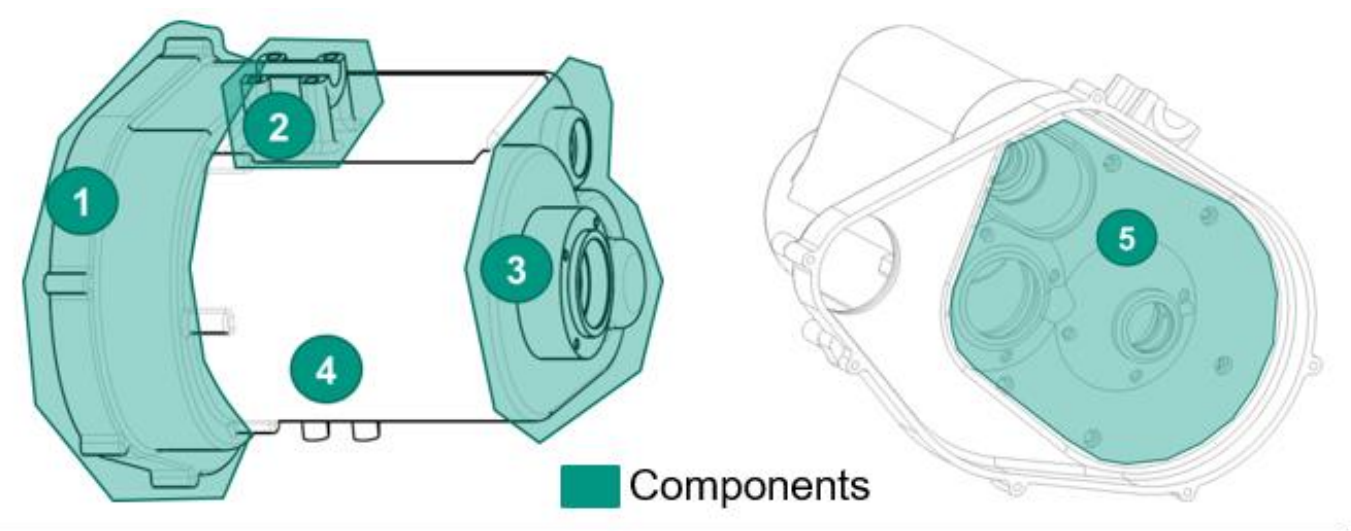

Figure 2. Exemplary gearbox housing according to Albers et al. (2018)

Table 4. Design readiness - TRL, IRL, cardinal DRL for three exemplary lightweight design gearbox housing concepts

\begin{tabular}{|c|c|c|c|c|c|c|}
\hline Component & Concept 1 & TRL & Concept 2 & TRL & Concept 3 & TRL \\
\hline 1 & steel casting & 9 & steel casting & 9 & steel casting & 9 \\
\hline 2 & steel casting & 9 & steel casting & 9 & steel casting & 9 \\
\hline 3 & aluminium casting & 7 & $\begin{array}{l}\text { FRP injection } \\
\text { moulding }\end{array}$ & 7 & $\begin{array}{l}\text { FRP injection } \\
\text { moulding }\end{array}$ & 7 \\
\hline 4 & aluminium casting & 7 & $\begin{array}{l}\text { FRP injection } \\
\text { moulding }\end{array}$ & 3 & aluminium casting & 7 \\
\hline 5 & steel casting & 9 & steel casting & 9 & steel casting & 9 \\
\hline & & & & & & \\
\hline $\begin{array}{l}\text { Joining technologies } \\
\text { between components } \\
i, j\end{array}$ & Concept 1 & IRL & Concept 2 & IRL & Concept 3 & IRL \\
\hline $3, \mathrm{~K} 2$ & $\begin{array}{l}\text { cylindrical press fit } \\
\text { steel - aluminium }\end{array}$ & 7 & inserts & 5 & inserts & 5 \\
\hline 1,4 & steel - aluminium & 7 & steel - FRP & 3 & steel - aluminium & 7 \\
\hline$\ldots$ & $\ldots$ & $\ldots$ & $\ldots$ & $\ldots$ & $\ldots$ & $\ldots$ \\
\hline 3,4 & aluminium - aluminium & 8 & FRP - FRP & 3 & aluminium - FRP & 3 \\
\hline $\begin{array}{l}\text { Estimated weight } \\
\text { reduction }\end{array}$ & \multicolumn{2}{|l|}{ low } & \multicolumn{2}{|l|}{ high } & \multicolumn{2}{|l|}{ medium } \\
\hline $\begin{array}{l}\text { Last technology to } \\
\text { reach max. TRL / } \\
\text { IRL }\end{array}$ & \multicolumn{2}{|l|}{$\begin{array}{l}\text { aluminium casting } \\
\text { (in about } 6 \text { months) }\end{array}$} & \multicolumn{2}{|c|}{$\begin{array}{l}\text { FRP injection moulding } \\
\text { (in about } 24 \text { months) }\end{array}$} & \multicolumn{2}{|l|}{$\begin{array}{l}\text { aluminium - FRP } \\
\text { (in about } 12 \text { Months) }\end{array}$} \\
\hline $\begin{array}{l}\text { DRL [based on TRL } \\
\& \text { IRL forecast] }\end{array}$ & \multicolumn{2}{|l|}{0.89} & \multicolumn{2}{|c|}{0.65} & \multicolumn{2}{|l|}{0.81} \\
\hline
\end{tabular}

For the gearbox housing components currently made of steel casting, components 3 and 4 are to be alternatively manufactured as aluminium casting or FRP injection moulding. The TRL corresponding to the choice of the manufacturing technology of a component and the IRL corresponding to the technological maturity of the joining technology (each on a scale of 1 to 9 ) are shown in Table 4.

FRP injection moulding has a lower TRL for component 4 than for component 3, demonstrating that FRP injection moulding has not yet been validated in laboratory environment for this kind of application.

In concept 2 and concept 3 , the use of FRP injection moulding in component 3 leads to the necessity to choose inserts as joining technology between the bearings (aggregated components K2) and component 3. For this, an IRL 5 is assumed. From the IRL's definition this means that the joining 
technology can be applied in principle, but no reliable statements about the suitability of the joining technology for this application are available yet.

If the concept to be pursued is chosen on the basis of potential weight saving, then concept 2 would be preferred. However, compared to the other concepts, this concept takes the longest time to be implemented (assuming that the same amount of work is put into all concepts). If a concept needs to be realized within a given timeframe, the last technology that can be implemented within a concept is the decisive one. By providing the predicted TRL and IRL, it becomes clear which critical technology (grey background in Table 4) is decisive for the timely implementation of the concept. In addition, the consideration of the cardinal DRL offers the possibility of estimating further workloads in the overall project. The cardinal DRL of 0.65 achieved in concept 2 reflects a concept maturity of $65 \%$.

Considering normalised values $D R L_{i}$ in addition to the DRL cardinal values, individual components which imply uncertainties for the implementation of a concept (Table 5) can be identified. Similar to the work of Garg et al. (2017), low TRL and IRL are assigned a greater uncertainty for the feasibility of the concept. According to Equation (4), $D R L_{i}$ represents both the maturity of the manufacturing technology $T R L_{i}$ and the maturity of the joining technologies $I R L_{i j}$ depending on $T R L_{j}$.

Table 5. Normalized SRL cardinal values for individual components

\begin{tabular}{|l|c||c|c|c|c|c|c|c|c|}
\cline { 2 - 9 } \multicolumn{1}{c|}{} & DRL & $D R L_{1}$ & $D R L_{2}$ & $D R L_{3}$ & $D R L_{4}$ & $D R L_{5}$ & $D R L_{K 1}$ & $D R L_{K 2}$ & $D R L_{K 3}$ \\
\hline Concept 1 & 0.89 & 0.92 & 0.88 & 0.82 & 0.82 & 0.87 & 1 & 0.86 & 1 \\
\hline Concept 3 & 0.81 & 0.92 & 0.88 & 0.46 & 0.69 & 0.87 & 1 & 0.68 & 1 \\
\hline
\end{tabular}

Although concept 3 has only a slightly lower DRL cardinal value than concept 1 , it is noticeable that, for example, $D R L_{3}$ is significantly lower than in concept 1 . Thus, there are greater uncertainties for the integration of component 3 into the overall concept. These uncertainties can also have an effect on the forecast of reaching series maturity. Moreover, the normalised $D R L_{i}$ can also be used to argue for a strategic distribution of financial resources in order to accelerate the progress in concept implementation.

\section{Conclusion and outlook}

Especially in early phases of product development, decisions which significantly influence the further product development process are made. These decisions are usually made with a high degree of uncertainty. In order to support product development teams in the decision-making process of selecting MMD concept ideas, this paper presents a method that helps to create comparable data bases for different concepts. For this purpose, a joint evaluation of the manufacturing (TRL) and joining technologies (IRL) required for the concept is carried out on the basis of their temporal development in the past up to the estimated date of series maturity. So, the calculated Design Readiness Level differs from the System Readiness Level by considering the temporal development prognosis of the used technologies. Therefore, it serves as decision basis for an objective evaluation of the current design maturity of different MMD concepts. The method systematically prepares the available data in such a way that the product developer can make a better decision under consideration of the compromises to be made. The method is also applicable to other fields outside the MMD as long as there are physical components to be manufactured and joined.

Future research work should focus on the additional prognosis of potential costs until a technology reaches series maturity. This would make it possible to evaluate concepts at an early stage in terms of time and money required to implement them. An additional way of evaluating a concept would be to define suitable criteria associated with the technical implementation of a concept. An example is the possible number of units that can be manufactured per company using a certain technology (depending on the individual constraints of a company). If the maximum achievable capacity is less than the required number of units, this would be an exclusion criterion for the technology. The cost per component can also be used as a further criterion. In order to improve the result of the prognosis for the maturity of a technology, further research can be conducted to develop a tool that considers uncertainties in the temporal extrapolation of the TRL and IRL. 


\section{Acknowledgement}

The research documented in this manuscript has been funded by the German Research Foundation (DFG) within the International Research Training Group "Integrated engineering of continuous-discontinuous long fiber reinforced polymer structures". The support by the German Research Foundation (DFG) is gratefully acknowledged.

\section{References}

Albers, A., Moeser, G. and Revfi, S. (2018), "Synergy Effects by using SysML Models for the Lightweight Design Method 'Extended Target Weighing Approach", Proceedings of the 28th CIRP Design Conference, Nantes, France, May 23-25, 2018, Elsevier, Amsterdam, pp. 434-439. https://doi.org/10.1016/j.procir.2018.02.025

Albers, A. et al. (2017), "Die frühe Phase der PGE - Produktgenerationsentwicklung", Proceedings of the Stuttgarter Symposium für Produktentwicklung 2017, Fraunhofer Verlag, Stuttgart, pp. 1-10.

Austin, M. and York, D. (2015), "System Readiness Assessment (SRA) an Illustrative Example", Procedia Computer Science, Vol. 44, Elsevier, Amsterdam, pp. 486-496. https://doi.org/10.1016/j.procs.2015.03.031

Conrow, E. (2011), "Estimating Technology Readiness Level Coefficients", Journal of Spacecraft and Rockets, Vol. 48, No. 1, American Institute of Aeronautics and Astronautics, Virginia USA, pp. 146-152. https://doi.org/10.2514/1.46753

DIN (2003), "DIN 8580:2003-09: Fertigungsverfahren - Begriffe, Einteilung”, DIN Deutsches Institut für Normung e.V., Berlin.

Fahimian, M. and Behdinan, K. (2017), "On characterization of technology readiness level coefficients for design", Proceedings of the 21st International Conference on Engineering Design (ICED17), Vancouver, Canada, August 21-25, 2017, The Design Society, Glasgow, pp. 309-316.

Garg, T. et al. (2017), "Using TRLs and system architecture to estimate technology integration risk", Proceedings of the 21st International Conference on Engineering Design (ICED17), Vancouver, Canada, August 21-25, 2017, The Design Society, Glasgow, pp. 301-310.

Gove, R., Sauser, B. and Ramirez Marquez, J. (2010), "Integration maturity metrics: Development of an integration readiness level”, Information Knowledge Systems Management, Vol. 9, No. 1, pp. 17-46. https://www.doi.org/10.3233/IKS-2010-0133

Mankins, J.C. (1995), Technology Readiness Levels. A White Paper. [online] Office of Space Access and Technology NASA. Available at: https://aiaa.kavi.com/apps/group_public/download.php/2212/TRLs_ MankinsPaper_1995.pdf (accessed 29.08.2019).

Mankins, J.C. (2009a), “Technology readiness and risk assessments: A new approach”, Acta Astronautica, Vol. 65 No. 9-10, Elsevier, Amsterdam, pp. 1208-1215. https://www.doi.org/10.1016/j.actaastro.2009.03.059

Mankins, J.C. (2009b), “Technology readiness assessments: A retrospective”, Acta Astronautica, Vol. 65 No. 910, Elsevier, Amsterdam, pp. 1216-1223. https://www.doi.org/10.1016/j.actaastro.2009.03.058

Matthiesen, S. et al. (2018), "From Reality to Simulation - Using the C\&C2-Approach to Support the Modelling of a Dynamic System", Proceedings of the 29th CIRP Design Conference, Póvoa de Varzim, Portugal, May 08-10, 2018, Elsevier, Amsterdam, pp. 475-480. http://dx.doi.org/10.1016/j.procir.2018.03.039

Saaty, T.L. (1990), "How to make a decision: The analytic hierarchy process", European Journal of Operational Research, Vol. 48 No. 1, pp. 9-26. https://www.doi.org/10.1016/0377-2217(90)90057-I

Sauser, B. et al. (2009), A systems approach to expanding the technology readiness level within Defense Acquisition. [online] School of Systems and Enterprises, Stevens Institute of Technology. Available at: https://apps.dtic.mil/docs/citations/ADA530242 (accessed 29.08.2019).

United States Government Accountability Office (2011), DEFENSE ACQUISITIONS Assessments of Selected Weapon Programs. U.S. Government Accountability Office. Available at https://apps.dtic.mil/dtic/tr/ fulltext/u2/a540208.pdf (accessed 11.11.2019). 
